\title{
Comparison of Treatment with Sitagliptin or Sulfonylurea in Patients with Type 2 Diabetes Mellitus and Mild Renal Impairment: A Post Hoc Analysis of Clinical Trials
}

\author{
Elizabeth S. Ommen • Lei Xu • Edward A. O'Neill • Barry J. Goldstein • \\ Keith D. Kaufman · Samuel S. Engel
}

To view enhanced content go to www.diabetestherapy-open.com

Received: November 17, 2014 / Published online: January 30, 2015

(c) The Author(s) 2015. This article is published with open access at Springerlink.com

\section{ABSTRACT}

Introduction: Impaired renal function is a major complication of type 2 diabetes mellitus (T2DM). Mild renal impairment is present in $38 \%$ of patients with T2DM and may impact choice of antihyperglycemic agent. Sulfonylureas and dipeptidyl peptidase-4 (DPP4) inhibitors are commonly used to treat hyperglycemia in patients with T2DM and renal impairment. Although in general sulfonylurea use is associated with an increased risk of hypoglycemia and weight gain, while DPP-4 inhibitor use is associated with a low risk of hypoglycemia, and is weight

Electronic supplementary material The online version of this article (doi:10.1007/s13300-015-0098-y) contains supplementary material, which is available to authorized users.

E. S. Ommen · L. Xu • E. A. O’Neill .

B. J. Goldstein - K. D. Kaufman - S. S. Engel

Merck \& Co., Inc., Whitehouse Station, NJ, USA

E. S. Ommen $(\bowtie)$

Clinical Research Department, Merck Research

Laboratories, RY34-A214, Rahway, NJ 07065, USA

e-mail: elizabeth.ommen@merck.com

Present Address:

B. J. Goldstein

Covance, Inc., Princeton, NJ, USA neutral, the relative efficacy and tolerability of these agents in patients with mild renal impairment has not been evaluated.

Methods: In a post hoc analysis, data from 1,211 subjects with T2DM and mild renal impairment (estimated glomerular filtration rates of 60 to $<90 \mathrm{~mL} / \mathrm{min} / 1.73 \mathrm{~m}^{2}$ ), who completed 25 or 30 weeks of one of three double-blind clinical trials comparing the DPP4 inhibitor sitagliptin $100 \mathrm{mg} /$ day with sulfonylureas in titrated doses, were pooled. The analysis compared change from baseline in glycated hemoglobin $\left(\mathrm{HbA}_{1 \mathrm{c}}\right)$, fasting plasma glucose (FPG), body weight, incidence of symptomatic hypoglycemia and the percentages of subjects meeting a composite endpoint of $\mathrm{HbA}_{1 \mathrm{c}}$ decrease $>0.5 \%$ without symptomatic hypoglycemia or body weight gain between sitagliptin and sulfonylurea treatment groups.

Results: $\mathrm{HbA}_{1 \mathrm{c}}$ and FPG decreased similarly with sitagliptin or sulfonylurea. A lower incidence of hypoglycemia was observed with sitagliptin. Body weight decreased with sitagliptin but increased with sulfonylurea. A greater percentage of subjects treated with sitagliptin (41.1\%) than treated with sulfonylurea (16.9\%) 
achieved the composite endpoint of $>0.5 \%$ $\mathrm{HbA}_{1 \mathrm{c}}$ reduction with no symptomatic hypoglycemia or body weight gain.

Conclusion: In this analysis of subjects with T2DM and mild renal impairment, treatment with sitagliptin provided glycemic efficacy similar to sulfonylurea, with less hypoglycemia and with body weight loss compared to body weight gain seen with sulfonylurea.

Trial Registrations: ClinicalTrials.gov \#NCT00482079, \#NCT00094770, \#NCT00701090.

Keywords: DPP-4; Hypoglycemia; Incretins; Renal impairment; Weight gain; Type 2 diabetes mellitus

\section{INTRODUCTION}

Among the many complications of type 2 diabetes mellitus (T2DM) arising from prolonged hyperglycemia, as well as from comorbid conditions such as hypertension, is chronic kidney disease (CKD), which is present in up to $40 \%$ of patients with T2DM [1]. Glycemic control reduces the incidence and progression of microvascular complications of diabetes [2,3], but achieving adequate glycemic control in patients with kidney disease can be challenging. This challenge may be underappreciated in patients with mild impairment in kidney function, who account for around 38\% of patients with T2DM [4].

The routine reporting of estimated glomerular filtration rate (eGFR) by clinical laboratories, most commonly using the Modification of Diet in Renal Disease (MDRD) equation [5], allows clinicians a greater awareness of patients' level of renal function and a greater recognition of even mild impairment. The introduction of sodium glucose co-transporter 2 inhibitors (SGLT2 inhibitors), a class of antihyperglycemic agent with a renal-based mechanism of action, has brought added emphasis to the importance of renal function in choice of treatment for T2DM. SGLT2 inhibitors demonstrate decreased glycemic efficacy as renal function declines, and this decrement begins in mild renal impairment [6]. Mild renal impairment may be relevant to metformin use as well: metformin is recommended as first-line therapy in most patients [7], but prescribing guidelines prohibit its use at specific thresholds of serum creatinine levels which correspond to only mild reductions in eGFR in some patients [8].

In the Fourth National Health and Nutrition Examination Survey (NHANES IV), sulfonylureas were the most commonly used antihyperglycemic agent in those with T2DM and mild CKD, though this was before the introduction of DPP4-inhibitors and other new agents [1]. Sitagliptin is a DPP4-inhibitor which, by preventing degradation of the incretins' glucagon-like peptide-1(GLP-1) and gastric inhibitory polypeptide (GIP), stimulates insulin release in a glucose-dependent manner [9]. Sitagliptin is approved for patients with all stages of CKD [10]; as sitagliptin is subject primarily to renal elimination [11], dose adjustment is recommended in patients with moderate-to-severe renal impairment or on dialysis so as to maintain drug exposure similar to that obtained in patients with normal renal function or only mild renal impairment [12]. A substantial number of large clinical studies have demonstrated sitagliptin to be generally safe and well tolerated [13]. In particular, because of the glucose-dependent nature of the insulinotropic effect of sitagliptin, the risk of hypoglycemia associated with use of this therapy is very low, except when used in combination with an agent that induces glucose-independent insulin secretion [14]. 
The efficacy and safety of sitagliptin vs. sulfonylurea in patients with T2DM and moderate-to-severe CKD or end-stage renal disease have been previously studied, showing similar glycemic efficacy between the two agents, and a lower risk of hypoglycemia and less weight gain with sitagliptin treatment, but otherwise generally similar safety and tolerability $[15,16]$. The current study is a post hoc analysis of data pooled from 3 clinical trials, comparing the efficacy, rates of hypoglycemia, and changes in body weight in patients with T2DM and mild renal impairment who were randomized to either sitagliptin or a sulfonylurea.

Subjects with mild renal impairment (eGFR of $\geq 60$ to $<90 \mathrm{~mL} / \mathrm{min} / 1.73 \mathrm{~m}^{2}$ ) were identified for this analysis using the MDRD equation to estimate glomerular filtration rate (GFR) [17]. In one of the 3 studies (referred to as Study 3), GFR could be estimated by both the MDRD and the Chronic Kidney Disease Epidemiology Collaboration (CKD-epi) serum creatinine [18] equations. The MDRD equation is imprecise and systematically underestimates GFR at higher levels of renal function [19]. Therefore, to evaluate the accuracy of classification of subject renal function by the MDRD equation (the only method available for Studies 1 and 2), baseline eGFR and renal function classification were evaluated by both equations for all subjects in Study 3 and compared.

\section{METHODS}

\section{Data Sources}

This was a post hoc analysis of pooled data from subjects who participated in one of three randomized, double-blind trials in which sitagliptin was compared to a sulfonylurea, had mild renal impairment (eGFR of $\geq 60$ to $<90 \mathrm{~mL} / \mathrm{min} / 1.73 \mathrm{~m}^{2}$ ) at baseline as determined by the 4 -variable MDRD equation [17] (meeting eGFR criteria for mild [Stage 2] CKD [20]), had glycated hemoglobin $\left(\mathrm{HbA}_{1 \mathrm{c}}\right)$ data at the analysis time points, and had no major protocol violations. There was no rescue therapy in these trials, as subjects with persistent hyperglycemia were discontinued.

In Study 1 (Sitagliptin Protocol 010, ClinicalTrials.gov\#NCT00482079) [21], 743 subjects were 21-76 years of age with baseline $\mathrm{HbA}_{1 \mathrm{c}}$ from 6.5 to $<10.0 \%$ on diet alone; for Study 2 (Sitagliptin Protocol 024, ClinicalTrials.gov\#NCT00094770) [22, 23], 1,172 subjects were $18-78$ years of age with baseline $\mathrm{HbA}_{1 \mathrm{c}}$ from 6.5 to $10.0 \%$ on background metformin monotherapy; and for Study 3 (Sitagliptin Protocol 803; ClinicalTrials.gov\#NCT00701090) [24], 1,034 subjects were $\geq 18$ years of age with baseline $\mathrm{HbA}_{1 \mathrm{c}}$ from 6.5 to $9.0 \%$ on background metformin monotherapy.

In Study 1, a dose-range finding study, subjects were randomized in an equal ratio among six treatment groups, of which two are appropriate for inclusion in the present analyses: the group randomized to sitagliptin (100 $\mathrm{mg}$ per day administered as $50 \mathrm{mg}$ twice daily) and the group randomized to glipizide (in titrated doses). The primary study duration was 12 weeks, subsequently extended to 104 weeks. In Study 2, subjects were randomized 1:1 to sitagliptin (100 mg per day) or glipizide (in titrated doses). Study duration was 104 weeks with the primary time point at Week 52. In Study 3, subjects were randomized 1:1 to sitagliptin (100 mg per day) or glimepiride (in titrated doses). Study duration was 30 weeks. In all 3 studies, sulfonylureas (or matching placebo) were titrated based on self-performed fingerstick glucose values. Up-titration was 
withheld if the investigator considered that uptitration would place the subject at unacceptable risk for hypoglycemia, and down-titration could be done at any time during the study to prevent recurrent hypoglycemic events. The mean \pm standard deviation dose of sulfonylurea achieved during the analysis period in subjects included in this analysis was $10.9 \pm 5.9 \mathrm{mg}$ glipizide per day, $10.4 \pm 6.5 \mathrm{mg}$ glipizide per day, and $2.0 \pm 1.4 \mathrm{mg}$ glimepiride per day in studies 1 , 2 , and 3, respectively.

As indicated above, the three studies had slight differences in their protocols, including background therapy (i.e., diet/exercise or metformin) and specific sulfonylurea comparator. However, efficacy and safety in both backgrounds are relevant to this analysis. Therefore, because it is not expected that a stable metformin background would impact body weight or hypoglycemic events, and because similar efficacy and side effect profiles of the two second-generation sulfonylureas were expected, data were combined from the three studies in order to increase the power and generalizability of the analysis.

All studies contributing data to the analysis reported here were conducted in accordance with principles of Good Clinical Practice and were approved by the appropriate institutional review boards and regulatory agencies. The analysis in this article is based on previously conducted studies, and does not involve any new studies of human or animal subjects performed by any of the authors.

\section{Analysis Endpoints}

Evaluation endpoints were changes from baseline in $\mathrm{HbA}_{1 \mathrm{c}}$, fasting plasma glucose (FPG), and body weight. The percentages of subjects with $\mathrm{HbA}_{1 \mathrm{c}}<7.0 \%$, the percentages of subjects with $\mathrm{HbA}_{1 \mathrm{c}}$ decrease $>0.5 \%$, and the percentages of subjects meeting a composite endpoint of $\mathrm{HbA}_{1 \mathrm{c}}$ decrease $>0.5 \%$ with no symptomatic hypoglycemia and no body weight gain at the end of the study periods were calculated. The percentages of subjects reporting at least one adverse event (AE) of symptomatic hypoglycemia, and the exposureadjusted event rate of reported AEs of symptomatic hypoglycemia that occurred during the analysis period were also calculated. An AE of symptomatic hypoglycemia was defined as any episode with symptoms consistent with hypoglycemia; there was no requirement for confirmation of hypoglycemia by a measurement of blood glucose levels. Severe hypoglycemia was defined as an episode of hypoglycemia that required assistance, either medical or nonmedical. Episodes with a markedly depressed level of consciousness, a loss of consciousness, or seizure were classified as having required medical assistance, whether or not medical assistance was obtained.

\section{Statistical Analysis}

In each study, the period evaluated began at first dose of sitagliptin or sulfonylurea and ended as close as possible to the final time point for the study of shortest duration (Week 30 of Study 3).For Study 1, Week 25 data were used for all endpoints. For Study 2, Week 30 data were used for $\mathrm{HbA}_{1 \mathrm{c}}$ and FPG and Week 24 data for body weight. For Study 3, Week 30 data were used for all endpoints. The incidence of hypoglycemia was assessed through Week 25 (Study 1) or 30 (Studies 2 and 3). Since treatment effects for both sitagliptin and sulfonylurea generally plateau by 6 months, these time points were considered to be similar with regard to the endpoints of 
interest. Further, any minor effects related to the 5 week difference would be equally balanced between the two treatment groups. Results at the time point used for this analysis, for each individual study for the full perprotocol analysis population, are provided in supplementary Table 1 .

To be included in the analysis population, subjects with appropriate eGFR at baseline had to have completed Study 1 through Week 25 or Studies 2 or 3 through Week 30, have a baseline

Table 1 Baseline demographic and anthropometric characteristics of randomized subjects in pooled study cohorts

\begin{tabular}{|c|c|c|}
\hline Parameter & $\begin{array}{l}\text { Sitagliptin } \\
(N=601)\end{array}$ & $\begin{array}{l}\text { Sulfonylurea } \\
(N=610)\end{array}$ \\
\hline Age (years) & $57.7 \pm 8.5$ & $57.6 \pm 9.4$ \\
\hline Male gender $[n(\%)]$ & $326(54.2)$ & $357(58.5)$ \\
\hline \multicolumn{3}{|l|}{$\operatorname{Race}[n(\%)]$} \\
\hline White & $428(71.2)$ & $416(68.2)$ \\
\hline Asian & $56(9.3)$ & $80(13.1)$ \\
\hline Black & $18(3.0)$ & $21(3.4)$ \\
\hline Other & $99(16.5)$ & $93(15.2)$ \\
\hline Body weight (kg) & $85.8 \pm 16.6$ & $86.4 \pm 16.8$ \\
\hline Body mass index $\left(\mathrm{kg} / \mathrm{m}^{2}\right)$ & $30.4 \pm 4.7$ & $30.8 \pm 4.8$ \\
\hline $\begin{array}{l}\text { Estimated GFR }[\mathrm{mL} / \mathrm{min} / \\
1.73 \mathrm{~m}^{2} \text { (interquartile } \\
\text { range) }]\end{array}$ & $\begin{array}{l}75.9 \pm 7.6 \\
(69.6,81.6)\end{array}$ & $\begin{array}{l}75.9 \pm 7.2 \\
\quad(70.4,81.3)\end{array}$ \\
\hline $\mathrm{HbA}_{1 \mathrm{c}}[\%$ (range) $]$ & $\begin{array}{l}7.6 \pm 0.8 \\
(6.1 \text { to } \\
10.5)\end{array}$ & $\begin{array}{c}7.6 \pm 0.9 \\
(5.8 \text { to } \\
11.0)\end{array}$ \\
\hline $\mathrm{FPG}(\mathrm{mg} / \mathrm{dL})$ & $154.9 \pm 35.9$ & $156.9 \pm 40.1$ \\
\hline Duration of T2DM (years) & $6.4 \pm 4.9$ & $5.8 \pm 4.5$ \\
\hline
\end{tabular}

Data are expressed as mean \pm standard deviation or frequency $[n(\%)]$

GFR glomerular filtration rate, FPG fasting plasma glucose, $T 2 D M$ type 2 diabetes mellitus. To convert FPG in $\mathrm{mg} / \mathrm{dL}$ to $\mathrm{mmol} / \mathrm{L}$ divide by 18 and end of analysis period $\mathrm{HbA}_{1 \mathrm{c}}$ measurement, and have no major protocol violations.

Analysis of covariance was used to compare the treatment group changes from baseline for the continuous endpoints, at time points indicated. The model controlled for treatment, study, and baseline value. The difference between treatment groups in change from baseline was assessed by testing the difference in the least squares mean change from baseline. Percentages and event rates were assessed using the method of Miettinen \& Nurminen [25], stratified by study to calculate a nominal $P$ value for between-group differences. The event rate was calculated as number of events divided by total subject-years of exposure. The total subject-years of exposure were calculated as the sum, over all subjects, of the time from the first dose to last dose of study medication for the time period included in this analysis.

Using all available data from Study 3, baseline eGFR and mild renal impairment classification using the 4-variable MDRD equation was compared with the baseline values and classification obtained using the CKD-epi creatinine equation, considering CKD-epi eGFR to be the reference value. All analyses were done using SAS (developed by SAS Institute Inc., Cary, NC, USA.), version 9.3.

\section{RESULTS}

Of the 1,538 randomized subjects with mild renal impairment across the three studies $(N=774$ treated with sitagliptin and $N=764$ treated with a sulfonylurea), 1,211 (78.7\%) met the criteria for inclusion in this analysis $[N=601(77.6 \%)$ treated with sitagliptin and $N=610(79.8 \%)$ treated with a sulfonylurea]. Baseline demographic and anthropometric characteristics of subjects in the two groups of the pooled study cohort were similar (Table 1). 
The mean age of subjects in the cohort was 57.7 years and their mean eGFR was $75.9 \mathrm{~mL}$ / $\min / 1.73 \mathrm{~m}^{2}$. The mean duration of T2DM in the cohort was 6.1 years.

Changes from baseline in $\mathrm{HbA}_{1 \mathrm{c}}$ were similar between the sitagliptin- and the sulfonylureatreated groups (sitagliptin $=-0.62 \%$, sulfonylurea $=-0.68 \%$; sitagliptin difference from sulfonylurea group $0.06 \%, P=0.104$; Fig. 1). The percentages of subjects with $\mathrm{HbA}_{1 \mathrm{c}}<7.0 \%$ or with $>0.5 \%$ reduction in $\mathrm{HbA}_{1 \mathrm{c}}$ were also similar between the two groups (differences from sulfonylurea group $-4.7 \%, P=0.092$, Fig. 2 and $0.2 \%, P=0.956$, Fig. 3, respectively). The FPG changes from baseline observed with sitagliptin treatment and with sulfonylurea treatment at Weeks 25 or 30 (Fig. 4) were similar (difference from sulfonylurea group $1.6 \mathrm{mg} / \mathrm{dL}, P=0.298$ ).

A significantly smaller percentage of subjects with mild renal impairment treated with sitagliptin reported experiencing at least one episode of symptomatic hypoglycemia compared with subjects treated with a sulfonylurea $(6.5 \%$ with sitagliptin vs. $25.9 \%$ with SU; $P<0.001 ;$ Fig. 5$)$. In addition, the reported number of symptomatic hypoglycemia events in the sitagliptin group

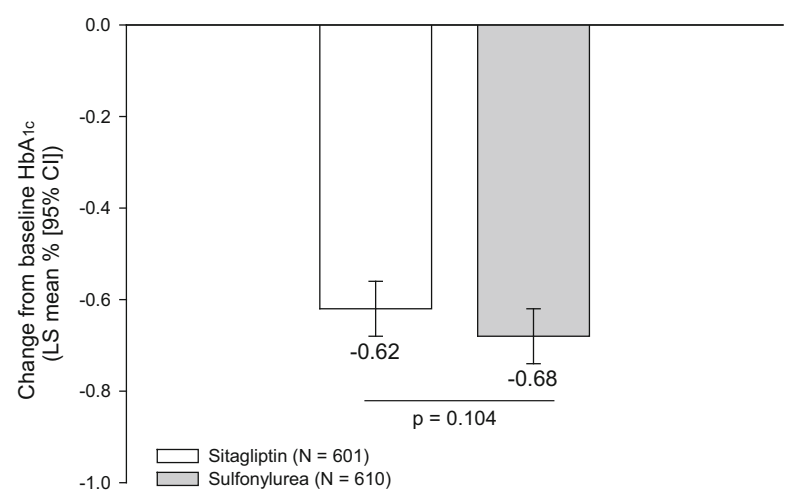

Fig. 1 Glycated hemoglobin $\left(\mathrm{HbA}_{1 \mathrm{c}}\right)$ change from baseline after 25-30 weeks of treatment with sitagliptin or a sulfonylurea. $C I$ confidence interval, $L S$ least squares

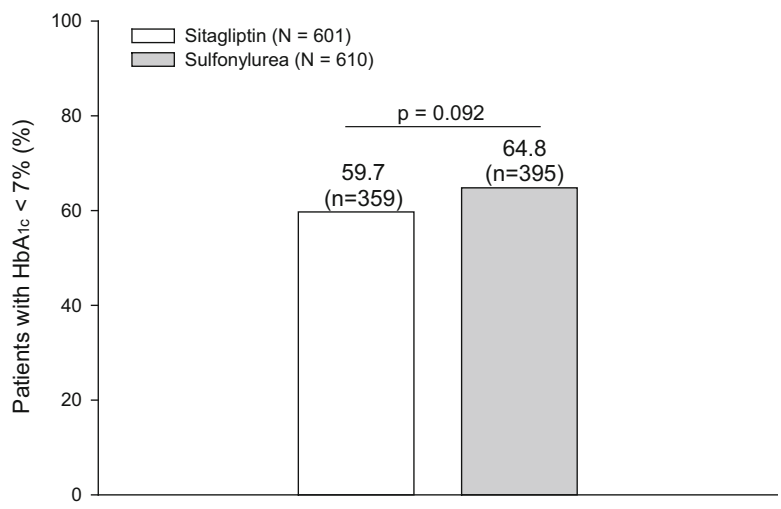

Fig. 2 Percentage of subjects with glycated hemoglobin $\left(\mathrm{HbA}_{1 \mathrm{c}}\right)<7.0 \%$ after $25-30$ weeks of treatment with sitagliptin or a sulfonylurea

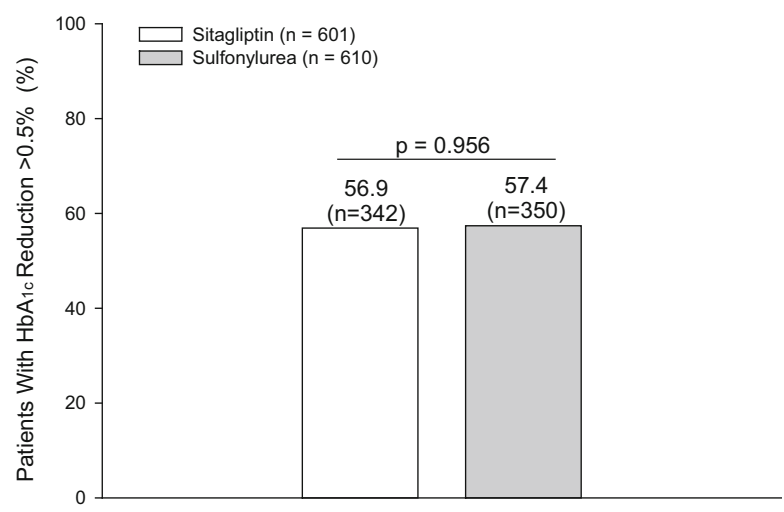

Fig. 3 Percentage of subjects with $>0.5 \%$ reduction in glycated hemoglobin $\left(\mathrm{HbA}_{1 \mathrm{c}}\right)$ after 25-30 weeks of treatment with either sitagliptin or a sulfonylurea

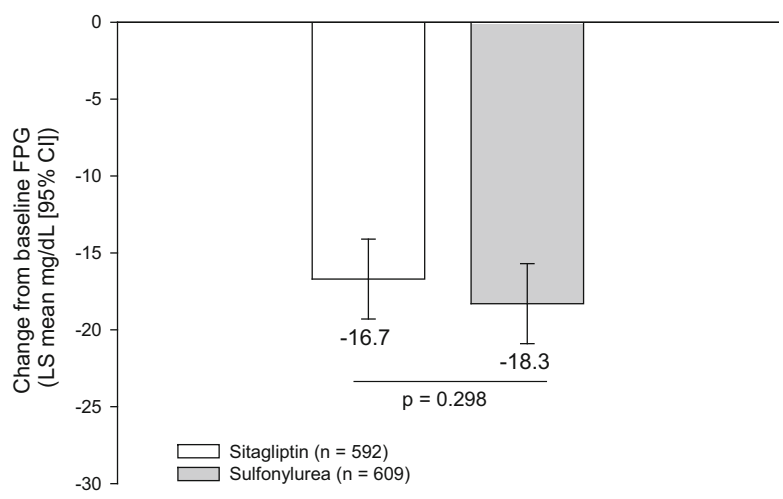

Fig. 4 Fasting plasma glucose (FPG) change from baseline after 25-30 weeks of treatment with sitagliptin or a sulfonylurea. $C I$ confidence interval, $L S$ least squares 


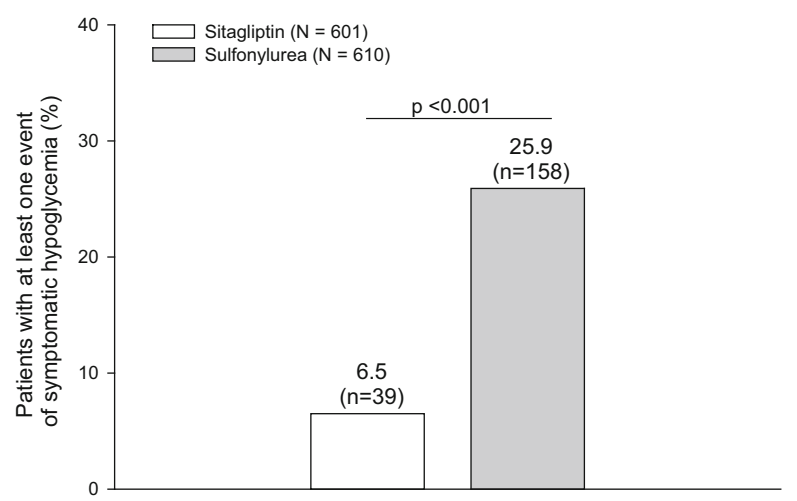

Fig. 5 Percentage of subjects with at least one episode of symptomatic hypoglycemia during treatment with sitagliptin or a sulfonylurea

during the analysis period (79) was lower compared to the sulfonylurea group (609), which, due to the similarity in treatment durations, led to a significantly lower event rate for symptomatic hypoglycemia in the sitagliptin group compared with the sulfonylurea group (Fig. 6). One subject in this cohort, treated with a sulfonylurea, reported at least one episode of severe hypoglycemia, while no subject treated with sitagliptin reported an event of severe hypoglycemia.

Mean body weight in the sulfonylurea group increased while that in the sitagliptin group decreased (Fig. 7), yielding a significant between-group difference of -2.3 kilograms $(P<0.001)$.

In the sitagliptin group, $41.1 \%$ of subjects achieved the composite endpoint of an $\mathrm{HbA}_{1 \mathrm{c}}$ decrease of $>0.5 \%$ with no reported event of symptomatic hypoglycemia and no increase in body weight, a substantially larger percentage than in the sulfonylurea group (between-group difference 24.6\%, $P<0.001$; Fig. 8).

In Study 3, kidney function was evaluated by both the standard MDRD equation and the more recently validated CKD-epi equation. A comparison of the results obtained by each method suggests that if the CKD-epi equation

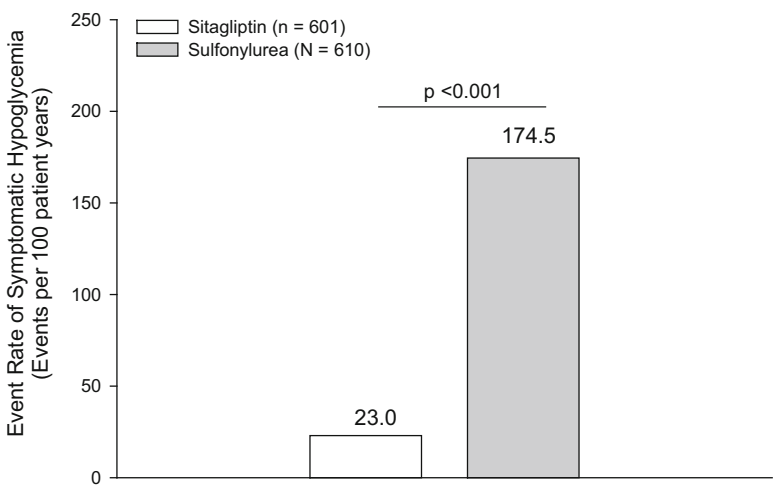

Fig. 6 Event rate of symptomatic hypoglycemia during treatment with sitagliptin or a sulfonylurea

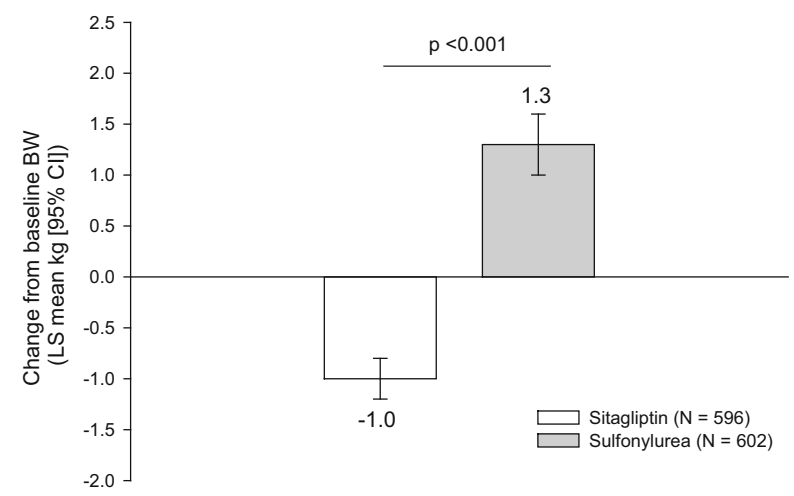

Fig. 7 Body weight (BW) change from baseline after 25-30 weeks of treatment with either sitagliptin or a sulfonylurea. $C I$ confidence interval, $L S$ least squares

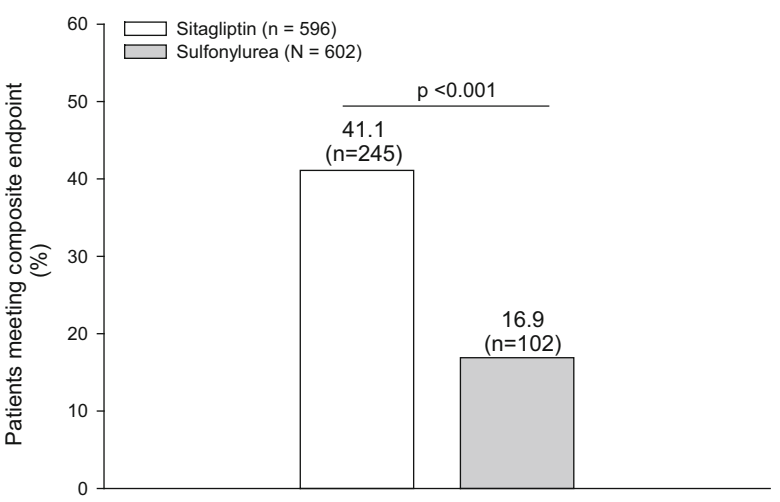

Fig. 8 Percentage of subjects meeting the composite endpoint of glycated hemoglobin $\left(\mathrm{HbA}_{1 \mathrm{c}}\right)$ decrease $>0.5 \%$ with no symptomatic hypoglycemia and no body weight gain after 25-30 weeks of treatment with either sitagliptin or a sulfonylurea 
Table 2 The glomerular filtration rates of all subjects in Study 3 were estimated using both the 4-variable Modification of Diet in Renal Disease (MDRD) equation and the Chronic Kidney Disease epidemiology collaboration (CKD-epi) creatinine equation

\begin{tabular}{lcccc}
\hline Subject eGFR classification & $<\mathbf{6 0}$ by MDRD & $\mathbf{2 6 0}$ to $<\mathbf{9 0}$ by MDRD & $>\mathbf{9 0}$ by MDRD & Total \\
\hline$<60$ by CKD-epi & $49(4.7)$ & $3(0.3)$ & $0(0.0)$ & $52(5.0)$ \\
60 to $\leq 89$ by CKD-epi & $6(0.6)$ & $489(47.2)$ & $16(1.5)$ & $511(49.4)$ \\
$>89$ by CKD-epi & $0(0.0)$ & $29(2.8)$ & $443(42.8)$ & $472(45.6)$ \\
Total & $55(5.3)$ & $521(50.3)$ & $459(44.3)$ & $1,035(100.0)$ \\
\hline
\end{tabular}

A comparison of the results of subject classification $[n(\%)]$ based on the results of each method is displayed

was used to classify subjects for the current pooled analysis, approximately $6.1 \%$ of subjects in the pooled analysis would not have met criteria for mild renal impairment (Table 2). Of 521 subjects classified by MDRD as having eGFR of 60 to $<90 \mathrm{~mL} / \mathrm{min} / 1.73 \mathrm{~m}^{2}, 3$ were classified by CKD-epi as having eGFR $<60 \mathrm{~mL} / \mathrm{min} /$ $1.73 \mathrm{~m}^{2}$ and 29 were classified by CKD-epi as having eGFR $\geq 90 \mathrm{~mL} / \mathrm{min} / 1.73 \mathrm{~m}^{2}$.

\section{DISCUSSION}

Tight glycemic control has been shown to reduce the risk of incident renal disease and to slow progression in patients with established renal disease [2, 3]. However, the rates of optimal glycemic control may be lower in patients with mild renal impairment compared to patients without renal disease or with more advanced disease. For example, in NHANES IV and in a large cohort of individuals at high risk for kidney disease, those individuals with T2DM who had mild CKD had significantly worse glycemic control compared to those who had no CKD or who had moderate or severe CKD [1, $26,27]$. The reasons for these findings are unclear, but may be due to lower levels of medical engagement by patients with mild renal impairment compared to those with more advanced renal impairment, or lower levels of vigilance by physicians when patients have only mild disease. This may be exacerbated by the practice of laboratories reporting MDRDderived eGFR calculations as " $\geq 60$ " for all individuals above that threshold, thus providing a sense of reassurance that may not be entirely warranted in patients with mild renal impairment.

While the choice of antihyperglycemic therapies for T2DM should take renal functional status into account, clinicians may not recognize mild renal impairment as a category requiring such consideration. Several clinical practice guidelines have recommended the use of the CKD-epi equation for estimating GFR, as the MDRD equation tends to underestimate the true GFR in those with higher GFR (e.g., mild renal impairment and normal renal function) $[28,29]$. However, in routine clinical practice, the majority of clinical laboratories continue to provide estimated GFR values using the MDRD equation [5, 28].

In this post hoc analysis of subjects with mild renal impairment, treatment with sitagliptin or sulfonylureas provided similar improvements in glycemic efficacy while there was a significantly higher incidence of AEs of symptomatic hypoglycemia with sulfonylurea compared with sitagliptin. Sulfonylurea treatment was also associated with weight gain compared with weight loss with sitagliptin. The results of this analysis show relative efficacy and 
tolerability in subjects with mild renal impairment identified by MDRD eGFR that is comparable to that obtained in the overall population from which these subjects were selected [21-24]. The overall findings of the analysis reported herein are attributable to the differing mechanisms of action of the two classes of drugs evaluated. Sulfonylureas are potent, glucose-independent insulin secretagogues which act directly on pancreatic beta cells [30]. Sulfonylureas stimulate a release of insulin in a glucose-independent fashion, making them effective antihyperglycemic agents and also increasing the likelihood of hypoglycemia. In contrast, the dipeptidyl peptidase-4 (DPP-4) inhibitor sitagliptin acts by stabilizing the DPP-4 substrates, GLP-1 and GIP, both incretin hormones which act in a glucose-dependent manner to increase the secretion of insulin by beta cells [9].

DPP-4 inhibitors are generally considered to be weight neutral, although the mild weight loss observed in several studies of broad populations of patients with T2DM [22-24], and in this analysis, may be related to stabilization of GLP-1; activation of the GLP-1 receptor with the GLP-1 agonist liraglutide has been shown to decrease body weight in patients with T2DM [31]. In contrast, weight gain with sulfonylureas is a known potential side effect which may result from increased insulin levels [32].

One limitation of this analysis is that AEs of hypoglycemia were reported based on symptoms and did not require measurement of blood glucose level. Another potential limitation is the use of the MDRD eGFR equation to identify the population with mild renal impairment for this analysis, as this equation has been shown to be less accurate at higher levels of renal function compared to the CKD-epi serum creatinine equation [18].
However, the comparison performed using data from all subjects in Study 3, in which both equations could be used, showed that the rate of possible misclassification was low, with approximately $94 \%$ of patients being accurately categorized using the MDRD equation. Moreover, as many clinical laboratories continue to use the MDRD equation to calculate eGFR whenever serum creatinine is performed $[5,28]$, the analysis reported here is relevant in real-world clinical practice settings.

Patients with diabetes and comorbid mild CKD represent a population at increased risk for progression to end-stage renal disease and for cardiovascular events compared to patients with diabetes without renal disease [33]. Management of hyperglycemia with minimization of side effects such as hypoglycemia and weight gain is clinically desirable in all patients with diabetes, but may be particularly important in patients with diabetes and $\mathrm{CKD}$, including mild $\mathrm{CKD}$, given the greater risks associated with this combination of diseases. From the current analysis, one can conclude that in patients with T2DM and mild renal impairment sitagliptin produces glycemic control similar to sulfonylureas but with substantially less hypoglycemia and without the weight gain generally observed with sulfonylureas.

\section{ACKNOWLEDGMENTS}

This analysis and its publication, including article processing fees, were funded by Merck \& Co., Inc., Whitehouse Station, NJ, USA. The authors acknowledge the contributions of Sheila Erespe at Merck\& Co., Inc. for assistance with submission of the article. All named authors meet the ICMJE criteria for authorship 
for this manuscript, take responsibility for the integrity of the work as a whole, and have given final approval to the version to be published.

Conflicts of interest. Elizabeth S. Ommen is an employee of Merck Sharp \& Dohme Corp., a subsidiary of Merck \& Co. Inc., Whitehouse Station, NJ, and may own shares of Merck \& Co., Inc. stock or stock options. Lei $\mathrm{Xu}$ is an employee of Merck Sharp \&Dohme Corp., a subsidiary of Merck \& Co. Inc., Whitehouse Station, NJ, and may own shares of Merck \& Co., Inc. stock or stock options. Edward A. O'Neill is an employee of Merck Sharp \& Dohme Corp., a subsidiary of Merck \& Co. Inc., Whitehouse Station, NJ, and may own shares of Merck \& Co., Inc. stock or stock options. At time of writing Barry J. Goldstein was an employee of Merck Sharp \& Dohme Corp., a subsidiary of Merck \& Co. Inc., Whitehouse Station, NJ, and may own shares of Merck \& Co., Inc. stock or stock options. Keith D. Kaufman is an employee of Merck Sharp \& Dohme Corp., a subsidiary of Merck \& Co. Inc., Whitehouse Station, NJ, and may own shares of Merck \& Co., Inc. stock or stock options. Samuel S. Engel is an employee of Merck Sharp \& Dohme Corp., a subsidiary of Merck \& Co. Inc., Whitehouse Station, NJ, and may own shares of Merck \& Co., Inc. stock or stock options.

Compliance with ethics guidelines. All studies contributing data to the analysis reported here were conducted in accordance with principles of Good Clinical Practice and were approved by the appropriate institutional review boards and regulatory agencies. The analysis in this article is based on previously conducted studies, and does not involve any new studies of human or animal subjects performed by any of the authors.
Open Access. This article is distributed under the terms of the Creative Commons Attribution Noncommercial License which permits any noncommercial use, distribution, and reproduction in any medium, provided the original author(s) and the source are credited.

\section{REFERENCES}

1. Koro CE, Lee BH, Bowlin SJ. Antidiabetic medication use and prevalence of chronic kidney disease among patients with type 2 diabetes mellitus in the United States. Clin Ther. 2009;31:2608-17.

2. Holman RR, Paul SK, Bethel MA, Matthews DR, Neil HA. 10-year follow-up of intensive glucose control in type 2 diabetes. N Engl J Med. 2008;359:1577-89.

3. Ismail-Beigi F, Craven T, Banerji MA, et al. Effect of intensive treatment of hyperglycaemia on microvascular outcomes in type 2 diabetes: an analysis of the ACCORD randomised trial. Lancet. 2010;376:419-30.

4. Bailey RA, Wang Y, Zhu V, Rupnow MF. Chronic kidney disease in US adults with type 2 diabetes: an updated national estimate of prevalence based on kidney disease: improving global outcomes (KDIGO) staging. BMC Res Notes. 2014;7:415.

5. Miller G Practices and recommendations for reporting estimated glomerular filtration rate (eGFR). College of American Pathologists website (2014). http://www.cap.org/apps/docs/committees/ chemistry/current_status_reporting_egfr2013.pdf. Accessed December 15, 2014.

6. Barnett AH, Mithal A, Manassie J, et al. Efficacy and safety of empagliflozin added to existing antidiabetes treatment in patients with type 2 diabetes and chronic kidney disease: a randomised, double-blind, placebo-controlled trial. Lancet Diabetes Endocrinol. 2014;2:369-84.

7. Inzucchi SE, Bergenstal RM, Buse JB, et al. Management of hyperglycemia in Type 2 diabetes: a patient-centered approach: position statement of the American Diabetes Association (ADA) and the European Association for the Study of Diabetes (EASD). Diabetes Care. 2012;35:1364-79.

8. Kajbaf F, Arnouts P, de Broe M, Lalau JD. Metformin therapy and kidney disease: a review of guidelines and proposals for metformin withdrawal around 
the world. Pharmacoepidemiol Drug Saf. 2013;10:1027-35.

9. Herman GA, Stein PP, Thornberry NA, Wagner JA. Dipeptidyl peptidase-4 inhibitors for the treatment of type 2 diabetes: focus on sitagliptin. Clin Pharmacol Ther. 2007;81:761-7.

10. Ramirez G, Morrison AD, Bittle PA. Clinical practice considerations and review of the literature for the use of DPP-4 inhibitors in patients with type 2 diabetes and chronic kidney disease. Endocr Pract. 2013;19:1025-34.

11. Herman GA, Stevens C, Van Dyck K, et al. Pharmacokinetics and pharmacodynamics of sitagliptin, an inhibitor of dipeptidyl peptidase IV, in healthy subjects: results from two randomized, double-blind, placebo-controlled studies with single oral doses. Clin Pharmacol Ther. 2005;78:675-88.

12. Bergman AJ, Cote $\mathrm{J}, \mathrm{Yi} \mathrm{B}$, et al. Effect of renal insufficiency on the pharmacokinetics of sitagliptin, a dipeptidyl peptidase-4 inhibitor. Diabetes Care. 2007;30:1862-4.

13. Engel SS, Round E, Golm GT, Kaufman KD, Goldstein BJ. Safety and tolerability of sitagliptin in type 2 diabetes: pooled analysis of 25 clinical studies. Diabetes Ther. 2013;4:119-45.

14. Oyer DS. The science of hypoglycemia in patients with diabetes. Curr Diabetes Rev. 2013;9:195-208.

15. Arjona Ferreira JC, Marre M, Barzilai N, et al. Efficacy and safety of sitagliptin versus glipizide in patients with type 2 diabetes and moderate-tosevere chronic renal insufficiency. Diabetes Care. 2013;36:1067-73.

16. Arjona Ferreira JC, Corry D, Mogensen CE, et al. Efficacy and safety of sitagliptin in patients with type 2 diabetes and ESRD receiving dialysis: a 54-week randomized trial. Am J Kidney Dis. 2013;61:579-87.

17. Levey AS, Bosch JP, Lewis JB, Greene T, Rogers $\mathrm{N}$, Roth D. A more accurate method to estimate glomerular filtration rate from serum creatinine: a new prediction equation. Modification of Diet in Renal Disease Study Group. Ann Intern Med. 1999;130:461-70.

18. Levey AS, Stevens LA, Schmid $\mathrm{CH}$, et al. A new equation to estimate glomerular filtration rate. Ann Intern Med. 2009;150:604-12.

19. Stevens LA, Coresh J, Feldman HI, et al. Evaluation of the modification of diet in renal disease study equation in a large diverse population. J Am SocNephrol. 2007;18:2749-57.
20. National Kidney Foundation. K/DOQI clinical practice guidelines for chronic kidney disease: evaluation, classification, and stratification. Am J Kidney Dis. 2002;39:S1-266.

21. Scott R, Wu M, Sanchez M, Stein P. Efficacy and tolerability of the dipeptidyl peptidase- 4 inhibitor sitagliptin as monotherapy over 12 weeks in patients with type 2 diabetes. Int J Clin Pract. 2007;61:171-80.

22. Nauck MA, Meininger G, Sheng D, Terranella L, Stein PP. Efficacy and safety of the dipeptidyl peptidase-4 inhibitor, sitagliptin, compared with the sulfonylurea, glipizide, in patients with type 2 diabetes inadequately controlled on metformin alone: a randomized, double-blind, non-inferiority trial. Diabetes Obes Metab. 2007;9:194-205.

23. Seck T, Nauck M, Sheng D, et al. Safety and efficacy of treatment with sitagliptin or glipizide in patients with type 2 diabetes inadequately controlled on metformin: a 2-year study. Int J Clin Pract. 2010;64:562-76.

24. Arechavaleta R, Seck T, Chen Y, et al. Efficacy and safety of treatment with sitagliptin or glimepiride in patients with type 2 diabetes inadequately controlled on metformin monotherapy: a randomized, double-blind, non-inferiority trial. Diabetes Obes Metab. 2011;13:160-8.

25. Miettinen $\mathrm{O}$, Nurminen M. Comparative analysis of two rates. Stat Med. 1985;4:213-26.

26. Whaley-Connell AT, Sowers JR, McFarlane SI, et al. Diabetes mellitus in CKD: kidney Early Evaluation Program (KEEP) and National Health and Nutrition and Examination Survey (NHANES) 1999-2004. Am J Kidney Dis. 2008;51:S21-9.

27. Whaley-Connell A, Sowers JR, McCullough PA, et al. Diabetes mellitus and CKD awareness: the Kidney Early Evaluation Program (KEEP) and National Health and Nutrition Examination Survey (NHANES). Am J Kidney Dis. 2009;53:S11-21.

28. National Clinical Guideline Centre: chronic kidney disease. National Clinical Guideline Centre website (2014). http://www.nice.org.uk/mwg-internal/de5 fs23hu73ds/progress?id=Ns+XUW+6h6\&dl. Accessed December 22, 2014.

29. KDIGO. Clinical practice guideline for the evaluation and management of chronic kidney disease. Kindney Int. 2012;3(2013):1-150.

30. Panten U, Schwanstecher M, Schwanstecher C. Sulfonylurea receptors and mechanism of sulfonylurea action. Exp Clin Endocrinol Diabetes. 1996;104:1-9. 
31. Niswender K, Pi-Sunyer X, Buse J, et al. Weight change with liraglutide and comparator therapies: an analysis of seven phase 3 trials from the liraglutide diabetes development programme. Diabetes Obes Metab. 2013;15:42-54.

32. Barnett AH. Complementing insulin therapy to achieve glycemic control. Adv Ther. 2013;30:557-76.
33. Ninomiya $T$, Perkovic V, de Galan BE, et al. Albuminuria and kidney function independently predict cardiovascular and renal outcomes in diabetes. J Am Soc Nephrol. 2009;20:1813-21. 